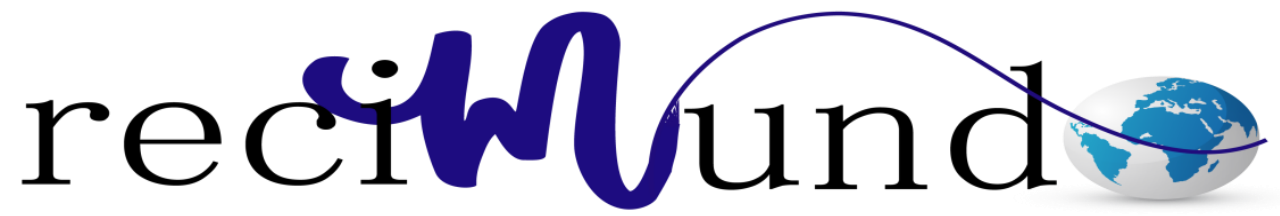

Revista Cientifica Mundo de la Investigación y el Conocimiento

Jahaira Paola Buñay Cantos ${ }^{\text {a }}$ J Jannina Alexandra Montalvan Espinoza ${ }^{\text {b; Sonia }}$ Elizabeth Vizueta León ${ }^{\mathrm{c}}$

Administración tradicional vs el Toyotismo: Una formula efectiva para las PyMES

Revista Científica Mundo de la Investigación y el Conocimiento. Vol. 2 núm., 1, febrero, ISSN: 2588-073X, 2018, pp. 464-487

DOI: $10.26820 /$ recimundo/2.1.2018.464-487

Editorial Saberes del Conocimiento

Recibido: 05/12/2017 Aceptado: 10/02/2018

a. Universidad de Guayaquil; jahaira.bunayca@ug.edu.ec

b. Universidad de Guayaquil; jannina.montalvanes@ug.com.ec

c. Universidad de Guayaquil; svizueta@ug.edu.ec 


\section{Administración tradicional vs el Toyotismo: Una formula efectiva para las}

PyMES

Vol. 2, núm. 1., (2018)

Jahaira Paola Buñay Cantos; Jannina Alexandra Montalvan Espinoza; Sonia Elizabeth Vizueta

León

\section{RESUMEN}

Las empresas en la actualidad requieren de personal altamente capacitado para lograr el éxito pretendido, principalmente de la posesión de un líder bien preparado en el ámbito de los negocios, que tenga la capacidad de dirigir la organización. Los administradores son las personas idóneas para realizar esta clase de actividades y buscar el logro de los objetivos organizacionales. Se llevó a cabo una investigación documental la cual permitió establecer una comparación entre el enfoque tradicional de administración versus el Toyotismo como una nueva tendencia integral en materia de administración de recursos para que así una PyMES se desempeñe exitosamente en el mundo global actual y futuro. Dicha investigación está basada en libros del área, los cuales dan sustento al tema desarrollado con ayuda de citas bibliográficas de los autores consultados; así como en internet como fuente de soporte.

Palabras claves: Administración, taylorismo, fordismo, toyotismo, PyMES. 


\title{
Administración tradicional vs el Toyotismo: Una formula efectiva para las PyMES
}

Vol. 2, núm. 1., (2018)

Jahaira Paola Buñay Cantos; Jannina Alexandra Montalvan Espinoza; Sonia Elizabeth Vizueta León

\begin{abstract}
Companies currently require highly trained personnel to achieve the intended success, mainly the possession of a well-prepared leader in the field of business, who has the ability to lead the organization. The administrators are the ideal people to carry out this kind of activities and look for the achievement of the organizational objectives. A documentary research was carried out which allowed us to establish a comparison between the traditional management approach versus Toyotism as a new integral trend in the administration of resources so that a SME can perform successfully in the current and future global world. This research is based on books from the area, which give support to the theme developed with the help of bibliographic citations of the authors consulted; as well as on the internet as a source of support.
\end{abstract}

Keywords: Administration, Taylorism, Fordism, Toyotism, PyMES. 


\section{Administración tradicional vs el Toyotismo: Una formula efectiva para las}

PyMES

Vol. 2, núm. 1., (2018)

Jahaira Paola Buñay Cantos; Jannina Alexandra Montalvan Espinoza; Sonia Elizabeth Vizueta

León

\section{Introducción.}

Una organización simboliza un factor clave en materia de generación de riqueza con la que cuenta una sociedad; lo cual permite el desarrollo económico y por consiguiente acceso a aspectos de bienestar para una comunidad. Desde el punto de vista tradicional, la empresa es considerada una entidad que organiza con eficiencia los factores económicos, produciendo bienes o servicios para satisfacer las necesidades del mercado y con ánimo de alcanzar ciertos objetivos, entre ellos el lucro y para alcanzar tales objetivos se debe contar con una administración eficiente de los recursos con los que cuenta dicha empresa.

Es por ello que la administración consiste en darle forma, de manera consistente y constante a las organizaciones, por medio del cumplimiento de metas que a su vez deben estar alineadas al plan estratégico que desee alcanzar la empresa y bajo un nuevo esquema que incluye a los trabajadores en ese proceso de alineación constante y evaluación del entorno, para establecer procesos flexibles que no impacten de manera negativa en la producción de un bien. Siendo todo lo anterior la base que impulsa la creación de un estudio bibliográfico que permita analizar groso modo la evolución de los enfoques de la administración desde su método más convencional hasta las nuevas tendencias que se han venido aplicado en los últimos tiempos para optimizar procesos productivos, relaciones laborales, así como aspectos de comercialización y finanza y como un nuevo método de trabajado puede apoyar en los procesos de creación de plusvalor en una compañía pese a lo pequeña que pueda ser. 


\section{Administración tradicional vs el Toyotismo: Una formula efectiva para las PyMES}

Vol. 2, núm. 1., (2018)

Jahaira Paola Buñay Cantos; Jannina Alexandra Montalvan Espinoza; Sonia Elizabeth Vizueta León

\section{Metodología y métodos}

\section{Antecedentes}

Las organizaciones en su dimensión de entes económicos y sociales son la base fundamental en donde se ve reflejado el crecimiento sostenido de una nación y por ende representa el progreso de una sociedad no solo en materia de riqueza, sino también de desarrollo de competencias técnicas, formación académica, reforzamiento o mejoramiento del sistema de valores de los ciudadanos, optimización de la productividad y evolución de un país en términos integrales, pero para que lo previamente mencionado tenga cabida las empresas deben contar con personas (trabajadores) que desempeñen una labor y a su vez con "un administrador" que se encargue de darle un uso óptimo a todos los recursos (económicos, financieros, técnicos, humanos, entre otros) para así lograr alcanzar los objetivos de rentabilidad para los accionistas de una empresa. En este sentido, la administración juega un papel indispensable y vital, pues es el eje conductor que articula todas las aristas que componen a una organización, los recursos con los que cuenta y da respuesta directa a la gestión del conocimiento que genera plusvalor y en consecuencia capitalización de una compañía.

La práctica administrativa contempla principalmente el negocio, pero su campo de acción no se limita exclusivamente en generar rentabilidad en una empresa, al ser una competencia exclusivamente humana, ésta se manifiesta en aspectos sociales, políticos, deportivo, religioso, doméstico, etc. De allí la importancia de visualizar a la administración como una actividad universal, e indistintamente de su área de ejecución, las personas que integran una organización (bien sea política, religiosa, lucrativa o de otra índole) deben trabajar en equipo en aras de 


\section{Administración tradicional vs el Toyotismo: Una formula efectiva para las}

PyMES

Vol. 2, núm. 1., (2018)

Jahaira Paola Buñay Cantos; Jannina Alexandra Montalvan Espinoza; Sonia Elizabeth Vizueta

León

alcanzar metas comunes, pero esto hace que dependiendo del administrador a cargo se establezcan matices en la ejecución de utilización de los recursos.

Es por ello que se hace vital definir el significado de la administración y su campo de acción, tarea nada sencilla, debido a que unos autores la entienden como una propiedad exclusiva de la empresa, otros la asumen como un proceso inherente a sus funciones en una organización, otros la consideran una ciencia, técnica o incluso un arte; siendo todas estas aproximaciones valederas, ya que en su momento dieron respuesta a un determinado momento de la sociedad y del tiempo en el cual se vivía, de allí que surjan tantos conceptos y enfoques, orientados al manejo eficiente de recursos, a medir tiempo para mejorar la productividad, establecer por medio de la repetición de actividades el condicionamiento de Pávlov en pro de alcanzar la efectividad y eficiencia en los procesos ejecutados, y bajo las nuevas tendencias propiciar valor agregado a través de la gestión del conocimiento, por medio de procesos racionales y nada autómatas.

Al ser la administración una actividad única y producto del ser humano, la misma tiende a ser manipulada por los rasgos y personalidad de quien la genera, por lo tanto, en ocasiones puede manifestarse como un concepto de arte, utilizando técnicas de motivación y liderazgo. Sin embargo, etimológicamente el origen de la palabra administración se forma del prefijo $a d$., hacia, y con ministrato, que proviene de minister, vocablo compuesto de minus, comparativo de inferioridad, y sufijo ter, que funge como termino de comparación. Por lo cual se puede concluir que minister a diferencia de magister (comparativo de superioridad) refleja un estado de inferioridad, subordinación y obediencia, por lo cual ad-ministrare, significa "prestar un servicio bajo el mando de otro" (Chiavenato, 2001) 


\section{Administración tradicional vs el Toyotismo: Una formula efectiva para las PyMES}

Vol. 2, núm. 1., (2018)

Jahaira Paola Buñay Cantos; Jannina Alexandra Montalvan Espinoza; Sonia Elizabeth Vizueta León

En virtud de lo anterior se puede definir en términos propios que la administración representa la actividad humana cuyo objetivo radica en lograr de forma eficiente la utilización de los recursos con los que cuenta la organización en pro de alcanzar de forma satisfactoria los objetivos de la empresa (capitalización y mayor rentabilidad) como de los individuos que coexisten en dicha institución.

En general, existen dos tipos de administración: la pública, que se refiere a la actividad gubernamental o del Estado, y tiene el propósito alcanzar sus objetivos para beneficio del país; y la privada, referente al sector privado (una empresa), cuyo fin es lograr un beneficio para asegurar su permanencia y su crecimiento. Para efectos de este estudio analítico y bibliográfico, el enfoque se dará únicamente en términos del sector privado.

\section{Algunas definiciones}

- Según (Ponce, 1992) señala algunos conceptos de otros autores referente al enfoque que estos le dan a la administración, los cuales se mencionan a continuación:

- E.F.L. Brech: Es un proceso social que lleva consigo la responsabilidad de planear y regular en forma eficiente las operaciones de una empresa, para lograr un propósito dado.

- Henry Fayol: Administrar es prever, organizar, mandar, coordinar y controlar. 


\section{Administración tradicional vs el Toyotismo: Una formula efectiva para las}

PyMES

Vol. 2, núm. 1., (2018)

Jahaira Paola Buñay Cantos; Jannina Alexandra Montalvan Espinoza; Sonia Elizabeth Vizueta

León

- José Antonio Fernández Arena: Es la ciencia social que persigue la satisfacción de objetivo instituciones por medio de la estructura y del esfuerzo humano coordinado.

- W. Jimenez Castro: Es una ciencia compuesta de principios, técnicas y prácticas cuya aplicación a conjuntos humanos permite establecer sistemas racionales de esfuerzos cooperativos, a través de los cuales se puede alcanzar propósitos comunes que individualmente no se puede lograr.

- Koontz y O’Donnell: Es la dirección de un organismo social, y su efectividad en alcanzar sus objetivos, fundada y habilidad de conducir a sus integrantes.

- J.D Mooney: Es el arte o técnica de dirigir e inspirar a los demás, con base en un producto y claro conocimiento de la naturaleza humana.

- Peterson y Plowman: Una técnica por medio de la cual se determinan, clarifican y realizan los propósitos y objetivos de un grupo humano particular.

- F. Tanneanbaum: El empleo de la autoridad para organizar, dirigir y controlar subordinados responsables, con el fin de que todos los servicios que se presentan san debidamente coordinados en el logro del fin de la empresa. 


\section{Administración tradicional vs el Toyotismo: Una formula efectiva para las PyMES}

Vol. 2, núm. 1., (2018)

Jahaira Paola Buñay Cantos; Jannina Alexandra Montalvan Espinoza; Sonia Elizabeth Vizueta León

- George Terry: Consiste en lograr un objetivo predeterminado mediante el esfuerzo ajeno". (p.68).

\section{Administración tradicional}

El enfoque de la administración tradicional se fundamenta en lo que hoy día se conoce como administración científica, la cual se basa en una confluencia de elementos que no existían en la antigüedad, ya que implica la coexistencia de valoración y manejo de conocimientos técnicos y leyes que afectan directamente a la organización, análisis del entorno, establecimiento y clasificación de competencias, reorientación de recursos, agrupados todos estos bajo un mismo escenario laboral, de forma tal de constituir la coordinación de los recursos en una ciencia que viene acompañada de un cambio de actitud recíproca de los trabajadores y de la alta gerencia; pues es el resultado de la división del trabajo y establecimiento de roles claros entre los actores que hacen vida dentro de la empresa para que la misma alcance sus objetivos.

De acuerdo a (Chiavenato, 2001) Taylor es el fundador del movimiento conocido como organización científica del trabajo. El pensamiento que lo guía es la eliminación de las pérdidas de tiempo, de dinero, etc., mediante un método científico. Afirma que "el principal objetivo de la administración debe ser asegurar el máximo de prosperidad, tanto para el empleador como para el empleado". Para el empleador, el máximo de prosperidad no significa la obtención de grandes beneficios a corto plazo, sino el desarrollo de todos los aspectos de la empresa para alcanzar un nivel de prosperidad. Para el empleado, el máximo de prosperidad no significa obtener grandes salarios de inmediato, sino un desarrollo personal para trabajar eficazmente, con calidad y utilizando sus dones personales. Taylor (Chiavenato, 2001) hace una 


\section{Administración tradicional vs el Toyotismo: Una formula efectiva para las}

PyMES

Vol. 2, núm. 1., (2018)

Jahaira Paola Buñay Cantos; Jannina Alexandra Montalvan Espinoza; Sonia Elizabeth Vizueta

León

distinción entre producción y productividad: "la máxima prosperidad es el resultado de la máxima productividad que, depende del entrenamiento de cada uno". Consciente de la oposición entre obreros y empleadores, da la siguiente explicación:

- Existe una falsa creencia, de que un aumento de la producción traerá el desempleo,

- Algunos malos sistemas de administración obligan al obrero a limitar su producción para proteger sus intereses pues, cuando él aumenta su ritmo de trabajo, el patrón se las arregla para no aumentarle su salario,

- Hay métodos de trabajo desastrosos que desperdician los esfuerzos de los obreros que reciben poca ayuda y pocos consejos por parte de la dirección.

El objetivo de la organización científica del trabajo (OCT) es derribar esos obstáculos y descubrir los métodos más eficaces para realizar una tarea y dirigir a los obreros: la "cooperación estrecha, íntima, personal, entre la administración y los obreros es la esencia misma de la OCT". "Lo que los trabajadores piden a sus empleadores es un salario elevado, y lo que los empleadores desean son bajos costos de producción la existencia o la ausencia de estos 2 elementos constituye el mejor indicio de una buena o de una mala administración".(p.128)

Taylor de acuerdo a (McGregor, 1960) enunció 4 principios de administración científica:

1. Estudio científico del trabajo, debe ser realizado por un equipo de especialistas; ese estudio dará lugar a la creación de una oficina o servicio de métodos de trabajo. Se definirán los procesos operativos más económicos y se establecerá la cantidad de trabajo que 


\section{Administración tradicional vs el Toyotismo: Una formula efectiva para las PyMES}

Vol. 2, núm. 1., (2018)

Jahaira Paola Buñay Cantos; Jannina Alexandra Montalvan Espinoza; Sonia Elizabeth Vizueta León

debe realizar un obrero colocado en condiciones óptimas; si el obrero obtiene esa cantidad deberá percibir un salario muy alto.

2. Selección científica y entrenamiento obrero. Taylor recomienda una selección sistemática según las aptitudes y estima que, cualquier trabajador puede resultar excelente para por lo menos un puesto de trabajo.

3. Unión del estudio científico del trabajo y de la selección científica del trabajador, "se trata de que los obreros apliquen la ciencia" y es allí donde fracasan muchos dirigentes y no los obreros, porque los primeros no quieren cambiar sus métodos,

4. Cooperación entre los dirigentes y los obreros; "el trabajo y la responsabilidad del trabajo se dividen de manera casi igual entre dirigentes y obreros". (p.128)

\section{Nuevas tendencias}

Luego de años de evolución y superada la revolución industrial queda instaurada la filosofía de Taylor y el fordismo, que no es más que un sistema socioeconómico basado en la producción industrial en serie. De acuerdo a (Chiavenato, 2001) Muchos estudiosos afirman que gran parte de los problemas se debe a las ingeniosas soluciones acuñadas a comienzos del siglo XX por dos ilustres estadounidenses, Frederic Taylor y Henry Ford, las cuales garantizaron con éxito la producción industrial masificada. Taylor fue el creador de lo que se convirtió en administración científica, un ordenamiento de los tiempos y ritmos en el proceso productivo. Con Taylor escéptico o frente a la eficiencia espontánea de los trabajadores, cuya indolencia es sistemática surgió la separación definitiva entre planeación, concepción y dirección del trabajo- 


\section{Administración tradicional vs el Toyotismo: Una formula efectiva para las}

PyMES

Vol. 2, núm. 1., (2018)

Jahaira Paola Buñay Cantos; Jannina Alexandra Montalvan Espinoza; Sonia Elizabeth Vizueta

León

que corresponde a la empresa y sus departamentos-, de la ejecución sencilla -que corresponde a los trabajadores-. El hombre gregario que debe obediencia a los instructores era el tipo ideal de obrero para Taylor. Esta concepción que era compartida por Ford, empresario convencido de la necesidad de precisión absoluta en la fabricación, quién afirmaba que "para ciertos hombres, la reproducción continua de una operación idéntica constituye una tarea horrible; para la mayoría, la repetición nada tiene de desagradable porque en muchos casos, la obligación de pensar es una verdadera tortura. Preocupado por la situación y tomando como modelo el transporte elevado en los mataderos de Chicago. Ford creó la primera línea de montaje en 1913 al introducir bandas transportadoras en su fábrica. Fue tal la subdivisión de tareas en el montaje de motores que nada menos que 85 obreros se encargaban de ejecutar el trabajo diseñado antes para un solo empleado. (p.51)

La diferencia del Fordismo con el taylorismo, es que lograron los objetivos no a costa del trabajador, sino que a través de una estrategia de expansión del mercado. (Coriat, 1991). Sin embargo, de acuerdo a (Soteldo \& al, 2005) el enfoque de la gestión del conocimiento recientemente ha resurgido como una perspectiva dominante que puede ser utilizada para explicar las dinámicas competitivas de los entornos cambiantes de la década de los noventas. Dicha perspectiva basada en los recursos ve a la empresa como un portafolio de competencias, las cuales son la fuente de ventajas competitivas sostenibles que residen en las competencias distintivas y en su habilidad para obtener beneficios de las mismas (Foss NJ, 1995). De acuerdo con (Porter, How information gives you competitive advantage, 1985) es a través de la dirección estratégica que se puede pasar de una orientación producto-mercado, a un contexto en el cual las 


\section{Administración tradicional vs el Toyotismo: Una formula efectiva para las PyMES}

Vol. 2, núm. 1., (2018)

Jahaira Paola Buñay Cantos; Jannina Alexandra Montalvan Espinoza; Sonia Elizabeth Vizueta León

empresas operen en entornos globales y donde se acelera el cambio tecnológico. En este nuevo contexto el enfoque producto-mercado encuentra limitaciones prácticas, por lo que actualmente existe un interés hacia los recursos internos de la propia empresa, específicamente hacia los recursos humanos.

(Soteldo \& al, 2005) señala que:

Así pues, el enfoque basado en los recursos y capacidades sugiere que los recursos que posee una empresa son más importantes que la manera en que está estructurada la industria a la que pertenece (Schendel, 1994). Para este enfoque estratégico, la ventaja competitiva de la empresa puede ser obtenida a través de las formas en que la organización configure y administre sus recursos internos que, cada vez más, serán una mayor fuente de ventajadiferencia entre las empresas. Según este enfoque, los diferentes desempeños en la empresa son el resultado de diferencias en la eficiencia, más que diferencias en el poder de mercado. Es así como, el conocimiento puede ser una fuente de ventaja competitiva sostenible y se fundamenta en dos propiedades importantes: primera, que está basado en la experiencia acumulada más que en la tarea o rutina organizativa, lo cual implica que contiene una gran cantidad de conocimiento tácito, lo que dificulta tanto su transferencia como su imitación por parte de los competidores; segunda, que no radica únicamente en los individuos, sino en la forma en que estos interactúan entre sí, es decir, el "capital social”, el cual resulta del trabajo en 


\section{Administración tradicional vs el Toyotismo: Una formula efectiva para las}

PyMES

Vol. 2, núm. 1., (2018)

Jahaira Paola Buñay Cantos; Jannina Alexandra Montalvan Espinoza; Sonia Elizabeth Vizueta

León

equipo en el que sus miembros poseen diferentes recursos de la organización

(Foss, Knuden y Montgomery, 1995). (p.31)

Dados los avances de la ciencia en todos los aspectos en los últimos 20 años, resurge un nombre que ha sido ícono en el mundo automotriz y ahora se ve reflejado en las nuevas tendencias administrativas del mundo modernos para cualquier empresa indistintamente de su tamaño, y es replicada su filosofía a nivel mundial, llamada el Toyotismo. Este nuevo enfoque de administración se produce en un momento en el cual el sistema capitalista buscaba cambios en sus procesos productivos, pues se encontraba en crisis, de allí nace lo que hoy día se entiende como neoliberalismo cuyos principios son:

- Estado mínimo de existencia de mercancía, no se busca sobre ofertar productos, sino ajustarlo a la demanda del mercado.

- Libre iniciativa tanto del empresariado como de los empleados, principalmente se auspicia la iniciativa en los niveles bajos de gerencia y cadenas productivas, en pro de desarrollar toma de decisiones asertivas y resolución de problemas antes situaciones operativas y/o complejas que no requiera del aporte estratégico de la alta gerencia, pues todos los miembros integrantes de una organización abocan sus esfuerzos en aras de alcanzar una meta en común.

- Todas las actividades se consideran mercancías De todos los sectores, el que más sufre con los cambios es la clase trabajadora, teniendo alteraciones en su estructura productiva, sindical y política. 


\section{Administración tradicional vs el Toyotismo: Una formula efectiva para las PyMES}

Vol. 2, núm. 1., (2018)

Jahaira Paola Buñay Cantos; Jannina Alexandra Montalvan Espinoza; Sonia Elizabeth Vizueta León

Desde la década de los 70, es palpable estos procesos de reestructuración para que las grandes potencias sigan manteniendo altos niveles de competitividad. En este contexto, se puede apreciar el patrón que hizo una reingeniería desde el punto de vista intelectual, de enfoque estratégico, de cultura organizacional, clima laboral, productividad y manejo de recursos, haciéndose merecedores de años de estudio y análisis en muchos países del mundo que hoy días buscan replicar este modelo, quienes apuestan a este enfoque de negocio especialmente en países en crisis o en vías de desarrollo por lo que comprende y lo que abarca más que un método de trabajo una filosofía empresarial que se alinea se termina alineados a la filosofía de vida de los trabajadores, en donde se aplica este enfoque. Según (Hoffman, 2012) Toyota y su modelo toyotista. A finales de los años 60, la compañía japonesa estaba plenamente dentro del modelo de producción flexible. Este modelo divulgado ampliamente dentro y fuera de Japón La ideología y los principios organizativos de este sistema comenzaron a mantener prácticas de negocio como un modelo de administración, siendo que en la década de 80 el Toyotismo pasó a ser la ideología universal de la producción sistémica del capital.

De acuerdo a (Hoffman, 2012):

El ingeniero japonés Eiji Toyoda visitó una industria automotriz en Detroit, dirigida por el sistema fordista, donde el flujo normal era producir primero y vender después. Fue entonces que, al evaluar la estructura de esta empresa, Toyoda se dio cuenta de que Japón no sería capaz de utilizar esa forma de producción. Por lo tanto, fue necesario modificar el sistema americano de producción. Buscando soluciones a 


\section{Administración tradicional vs el Toyotismo: Una formula efectiva para las}

PyMES

Vol. 2, núm. 1., (2018)

Jahaira Paola Buñay Cantos; Jannina Alexandra Montalvan Espinoza; Sonia Elizabeth Vizueta

León

este paradigma, Toyoda y su especialista en producción Taichi Ohno, iniciaron un proceso de cambios en la producción. Entre las nuevas técnicas implantadas, se encuentra la posibilidad de modificar máquinas rápidamente durante la producción, ampliando así la variedad de productos en oferta. Esa pasó a ser la esencia del modelo japonés de producción. Algunas reglas fueron implantadas y conforman las características del Toyotismo, partiendo del principio de que aquellos elementos que no agregasen valor al producto debían ser eliminados, como el tiempo perdido por reparaciones o material de desecho producción mayor de lo necesario, o antes del tiempo necesario operaciones innecesarias en el proceso de fabricación, transporte, stock del producto, movimiento humano, entre otros opera sobre la base de estos principios, el modelo de producción fue diseñado por: Automatización, Just-in-time, Trabajo en equipo, Manejo del estrés, Flexibilización de la mano de obra, Gestión participativa, Control de calidad (ISO-9000), tercerización de los aspectos no medulares de la organización que pudiesen disminuir de manera sustancial los costos de producción.

\section{Resultados.}

La filosofía Toyota aporta un nuevo enfoque para las empresas y sus accionistas, quienes a su vez dirigen sus esfuerzos a mantener márgenes aceptables de rentabilidad, en costos 


\section{Administración tradicional vs el Toyotismo: Una formula efectiva para las PyMES}

Vol. 2, núm. 1., (2018)

Jahaira Paola Buñay Cantos; Jannina Alexandra Montalvan Espinoza; Sonia Elizabeth Vizueta León

operativos prudentes, pero en especial se persigue dejar un legado en la industria, que va más allá de hacer branding (establecimiento de la marca en el mercado) y crear un vinculo afectivo de por vida con el trabajador hoy día es visto como un colaborador en el cumplimento de esa meta en común. Es por ello que se hace prudente presentar un cuadro comparativo entre el enfoque tradicional de la administración y las nuevas tendencias de estas, donde predomina en la actualidad en modelo toyotista.

\section{Cuadro $\mathrm{N}^{\circ}$ 1.- Comparativo de tendencias convencionales vs modernas de la} administración.

\begin{tabular}{|c|c|c|c|}
\hline \multirow{3}{*}{ ASPECTOS COMPARADOS } & \multicolumn{3}{|c|}{ ENFOQUES ADMINIS TRATIVOS } \\
\hline & \multicolumn{2}{|c|}{ TRADICIONAL } & \multirow{2}{*}{$\begin{array}{l}\text { MODERNA } \\
\text { Toyotismo }\end{array}$} \\
\hline & Taylorismo & Fordismo & \\
\hline OBJETIVOS & $\begin{array}{l}\text { Aumento de la productividad } \\
\text { Maximizar la eficiencia de la mano } \\
\text { de obra } \\
\text { Mayor control de los tiempo de } \\
\text { producción }\end{array}$ & $\begin{array}{l}\text { Producción masiva de productos } \\
\text { estandarizados } \\
\text { Flujo continuo y produccioón en } \\
\text { serie } \\
\text { Aumento de la división del trabajo }\end{array}$ & $\begin{array}{l}\text { Producir a bajos costos pequeñas cantidades } \\
\text { Producir varios tipos de productos }\end{array}$ \\
\hline BASES & $\begin{array}{l}\text { Método científico } \\
\text { Orientación positivista } \\
\text { Orientación mecanicista }\end{array}$ & $\begin{array}{l}\text { Producción en cadena } \\
\text { Organización altamente } \\
\text { especializada }\end{array}$ & $\begin{array}{l}\text { Producción en cadena } \\
\text { Método Japones Cinco (5) Ceros: } \\
\text { Cero Error, Cero Averías, Cero Demoras, Cero Papel, } \\
\text { Cero Existencias }\end{array}$ \\
\hline TIPO DE PRODUCCIÓN & En Masa & En Masa & A partir de los pedidos hechos a la fábrica \\
\hline MANO DE OBRA & $\begin{array}{l}\text { Pago a destajo de acuerdo a la } \\
\text { producción } \\
\text { Sistema de motiviación (pago de } \\
\text { primas) } \\
\text { Mayor es pecialización }\end{array}$ & $\begin{array}{l}\text { Salario alto y fijo } \\
\text { Trabajador más calificado }\end{array}$ & $\begin{array}{l}\text { Personal flexible } \\
\text { Ocupa varios roles } \\
\text { Trabajo en equipo }\end{array}$ \\
\hline VENTAJAS & $\begin{array}{l}\text { Mayor eficiencia de las personas } \\
\text { Disminuye la presión sobre un solo } \\
\text { jefe } \\
\text { División del trabajo }\end{array}$ & $\begin{array}{l}\text { Precios más bajos } \\
\text { Ritmo continuo de trabajo } \\
\text { Se empezó a introducir la } \\
\text { tecnología }\end{array}$ & $\begin{array}{l}\text { Implemento de la automatización / Control de calidad } \\
\text { Ahorro de tiempo } \\
\text { Bajos costos }\end{array}$ \\
\hline DESVENTAJAS & $\begin{array}{l}\text { Baja motivación y satisfacción } \\
\text { Baja calidad de producción } \\
\text { Alto aus entismo del personal }\end{array}$ & $\begin{array}{l}\text { Tiem po de ocio/ producción de un } \\
\text { solo tipo de producto } \\
\text { Trabajo repetitivo y ausente de } \\
\text { creatividad } \\
\text { Emplear menor cantidad de mano } \\
\text { de obra }\end{array}$ & $\begin{array}{l}\text { Productos variados } \\
\text { Horarios prolongados } \\
\text { Personal reducido }\end{array}$ \\
\hline LO QUE SOLVENTA & $\begin{array}{l}\text { Control de los trabajadores } \\
\text { Programación efectiva y eficiente de } \\
\text { las tareas } \\
\text { Precision de los costos de } \\
\text { producción }\end{array}$ & $\begin{array}{l}\text { Falta de habilidades en el personal } \\
\text { Simplificación de la organización } \\
\text { del trabajo } \\
\text { Reducción de los tiempo de } \\
\text { fabricación }\end{array}$ & $\begin{array}{l}\text { Intensificación del trabajo } \\
\text { Permite prescindir de Almacen (Cero Stock) } \\
\text { Precios de los productos } \\
\text { Realización de actividades variadas }\end{array}$ \\
\hline
\end{tabular}

Fuente: Elaboración propia, 2018. 


\section{Administración tradicional vs el Toyotismo: Una formula efectiva para las}

PyMES

Vol. 2, núm. 1., (2018)

Jahaira Paola Buñay Cantos; Jannina Alexandra Montalvan Espinoza; Sonia Elizabeth Vizueta

León

Si se observa posteriormente el detalle de la pequeña y mediana empresa en Ecuador, que de acuerdo a (Barrera, 2001) señala que:

En Ecuador, de acuerdo a su tamaño, las empresas tienen las categorías siguientes:

- Microempresas: emplean hasta 10 trabajadores, y su capital fijo (descontado edificios y terrenos) puede ir hasta 20 mil dólares.

- Talleres artesanales: se caracterizan por tener una labor manual, con no más de 20 operarios y un capital fijo de 27 mil dólares.

- Pequeña Industria: puede tener hasta 50 obreros

- Mediana Industria: alberga de 50 a 99 obreros, y el capital fijo no debe sobrepasar de 120 mil dólares.

- Grandes Empresas: son aquellas que tienen más de 100 trabajadores y 120 mil dólares en activos fijos

\section{Características}

El número de empresas y su la participación en la generación de empleo, se resume así: 


\section{Administración tradicional vs el Toyotismo: Una formula efectiva para las PyMES}

Vol. 2, núm. 1., (2018)

Jahaira Paola Buñay Cantos; Jannina Alexandra Montalvan Espinoza; Sonia Elizabeth Vizueta León

\section{Cuadro $N^{\circ}$ 2.- Empresas PYMES en Ecuador}

\begin{tabular}{cccc} 
SECTOR & $\begin{array}{c}\text { NUMERO } \\
\text { EMPRESAS }\end{array}$ & $\begin{array}{c}\text { PROMEDIO } \\
\text { EMPLEADOS } \\
\text { POR EMPRESA }\end{array}$ & $\begin{array}{c}\text { TOTAL } \\
\text { TRABAJADORES }\end{array}$ \\
PYMIS & 15.000 & 22 & 330.000 \\
Artesanías & 200.000 & 3 & 600.000 \\
Microempresas & 252.000 & 3 & 756.000 \\
\hline TOTAL & $\mathbf{4 6 7 . 0 0 0}$ & & $\mathbf{1 ' 6 8 6 . 0 0 0}$ \\
\cline { 2 - 2 } & & & \\
\hline
\end{tabular}

Fuente: (Barrera, 2001)

Ubicación geográfica: se da una gran concentración en las ciudades de mayor desarrollo; en Quito y Guayaquil se asientan el $77 \%$ de los establecimientos; en Azuay, Manabí y Tungurahua el 15\%; y el 8\% corresponde a 17 provincias.

Las principales diferencias en el sector son:

Cuadro $N^{\circ}$ 3.- Principales diferencias de la industria en Ecuador

\begin{tabular}{|cccc} 
& $\begin{array}{c}\% \\
\text { DE ESTABLE- } \\
\text { CIMIENTOS }\end{array}$ & $\begin{array}{c}\% \\
\text { PERSONAL } \\
\text { OCUPADO }\end{array}$ & $\begin{array}{c}\text { PIB } \\
\text { MILLONES } \\
\text { DOLARES }\end{array}$ \\
\hline PYMES & 84.3 & 37.7 & 458.8 \\
& & & $\begin{array}{c}24 \% \text { PIB } \\
\text { Manufactura }\end{array}$ \\
\hline GRAN INDUSTRIA & 15.7 & 62.3 & 1.371
\end{tabular}

Fuente: (Barrera, 2001) 


\section{Administración tradicional vs el Toyotismo: Una formula efectiva para las}

PyMES

Vol. 2, núm. 1., (2018)

Jahaira Paola Buñay Cantos; Jannina Alexandra Montalvan Espinoza; Sonia Elizabeth Vizueta

León

- La brecha de la productividad del trabajo entre la pequeña y gran industria se amplía. En 1988 el índice es de 0.876 y en 1990 baja al 0.398.

- Baja productividad hora/trabajador: Ecuador 3.25 dólares; Estados Unidos 30 dólares; Colombia entre 10 y 15 dólares.

- La competitividad de las PYMES ecuatorianas en el Grupo Andino es: menor respecto a Colombia y Venezuela, y mayor sobre el resto de países.

Indicadores: La situación actual de la pequeña industria se resume a continuación:

- Escaso nivel tecnológico

- Baja calidad de la producción, ausencia de normas y altos costos

- Falta de crédito, con altos cosos y difícil acceso

- Mano de obra sin calificación

- Producción se orienta más al mercado interno

- Incipiente penetración de PYMES al mercado internacional

- Ausencia total de políticas y estrategias para el desarrollo del sector

- Son insuficientes los mecanismos de apoyo para el financiamiento, capacitación, y uso de tecnología

- El marco legal para el sector de la pequeña industria es obsoleto

Potencialidades: La pequeña industria ecuatoriana cuenta con un sin número de potencialidades que son poco conocidas y aprovechadas. Principalmente se refieren a:

- Son factores claves para generar riqueza y empleo 


\section{Administración tradicional vs el Toyotismo: Una formula efectiva para las PyMES}

Vol. 2, núm. 1., (2018)

Jahaira Paola Buñay Cantos; Jannina Alexandra Montalvan Espinoza; Sonia Elizabeth Vizueta León

- Al dinamizar la economía, diluye los problemas y tensiones sociales, y mejorar la gobernabilidad.

- Requiere menores costos de inversión

- Es el factor clave para dinamizar la economía de regiones y provincias deprimidas

- Es el sector que mayormente utiliza insumos y materias primas nacionales

- Tiene posibilidades de obtener nichos de exportación para bienes no tradicionales generados en el sector

- El alto valor agregado de su producción contribuye al reparto más equitativo del ingreso

- Mantiene alta capacidad para proveer bienes y servicios a la gran industria (subcontratación)

- Es flexible para asociarse y enfrentar exigencias del mercado (p.1).

En virtud de lo anterior se puede observar que las pequeñas y medianas empresas en el Ecuador son un motor importante dentro de la economía de la nación, que venían con un porcentaje de participación importante dentro del PIB del mencionado país y por políticas estadas y falta de dinamismo de los empresarios comenzó un proceso de desaceleración que puede revertirse fácilmente. Al ser las PyMES una pieza angular dentro de la generación de fuentes de empleos y de medición de sostenibilidad de un país, es por lo que cual se invita al empresariados ecuatoriano a valerse de cualquier recurso que les permita optimizar la calidad de sus productos, los tiempos de entrega y el tipo de vínculo laboral que establecen con sus trabajadores, es por ello que si solamente el empresario busca aplicar nuevos métodos 


\section{Administración tradicional vs el Toyotismo: Una formula efectiva para las}

PyMES

Vol. 2, núm. 1., (2018)

Jahaira Paola Buñay Cantos; Jannina Alexandra Montalvan Espinoza; Sonia Elizabeth Vizueta

León

administrativos que no requiera de mantener altos niveles de inventario, inversiones mesuradas, poco personal pero eficiente y flexible para ejecutar diferentes roles dentro de la organización, generando que la competencia y el benchmarking se tras polen a los procesos internos de la empresa, propiciando así mejoramiento en la calidad y adaptabilidad al entorno comercial que rodea a la organización. Es por ello que se recomienda como una solución la aplicación del toyotismo en las PyMES de Ecuador.

Entendiendo que ningún proceso puede darse de manera aislada y sin el apoyo de todos los factores que confluyen para que materialice este esquema de trabajo, lo que incluye al Gobierno de Ecuador, la cámara de empresarios, el Ministerio de Educación Superior y los ciudadanos en general, pues esto implica un cambio no solo en el enfoque del negocio de una organización, sino en la restructuración de una filosofía de vida para el ecuatoriano, que fácilmente se puede convertir en un talento humano digno de exportación, así como la empresa donde labora y el producto que contribuye a fabricar.

\section{Conclusiones.}

La administración cumple con el objeto de manejar de la forma más eficiente los recursos de una empresa, entendiendo que dichos recursos pueden ser humanos, financieros, materiales, tecnológicos, de conocimientos o la combinación de todos los aspectos anteriores, entre otros. Sin embargo para cumplir con el fin propuesto por la compañía, la administración debe obedecer a un plan estratégico, a través de la ejecución de diversas técnicas y prácticas administrativas que propicien la organización, integración, dirección y control de los recursos para producir el beneficio establecido por la empresa, que bien puede ser social o económico, en el caso último se 


\section{Administración tradicional vs el Toyotismo: Una formula efectiva para las PyMES}

Vol. 2, núm. 1., (2018)

Jahaira Paola Buñay Cantos; Jannina Alexandra Montalvan Espinoza; Sonia Elizabeth Vizueta León

debe manifestar en términos monetarios para alcanzar altos niveles de rentabilidad, reflejando así el éxito de la misma. En este sentido, el toyotismo vino para perfeccionar un método buscando solucionar un paradigma: cómo ajustar el sistema de producción a los moldes neoliberales.

Por lo tanto, el Toyotismo representa la forma eficaz de administrarte ante los cambios de una forma innovadora y eficiente. Este sistema contempla al obrero especializado / trabajador convertido en colaborador dentro del proceso productivo bajo un nuevo enfoque de participación y toma de decisiones, ajustable a los cambios del entorno y midiéndose de acuerdo a los sistemas de gestión y control de la calidad con la premisa de Just In Time o Justo a tiempo en toda la cadena de suministro esté integrada verticalmente la empresa o no, es decir, que no se limita exclusivamente al inventario.

Al ser la pequeña y mediana empresa un elemento clave dentro del proceso productivo y de crecimiento sostenible de Ecuador, se hace indispensable que se sume a las nuevas tendencias mundiales, que a su vez le va a permitir expandir su manejo estructural, operativo y financiero, así como contribuirá a la optimización y posible diversidad de los productos que comercialice la empresa, estableciendo una nueva cultura organizacional, donde el establecimiento de vínculos afectivos con los trabajadores es vital para lograr alcanzar objetivos de rentabilidad y posible exportación de los productos fabricados a bajo costo.

\section{Bibliografía.}

Barrera, M. (2001). Situación y Desempeño de las PYMES de Ecuador en el Mercado Internacional recuperado

de: www.aladi.org/nsfaladi/reuniones.nsf/.../\$FILE/ecuador.doc. 


\section{Administración tradicional vs el Toyotismo: Una formula efectiva para las}

PyMES

Vol. 2, núm. 1., (2018)

Jahaira Paola Buñay Cantos; Jannina Alexandra Montalvan Espinoza; Sonia Elizabeth Vizueta

León

Chiavenato, I. (2001). Administración: Teoría, proceso y práctica. Mexico: McGraw-Hill Interamericana S.A.

Coriat, B. (1991). Coriat, B. (1991). El taller y el cronómetro. Ensayo sobre el taylorismo, el fordismo y la producción en masa, S XXI, México.

De freitas Ribeiro, A. (n. ed). Taylorismo, fordismo y toyotismo. recuperado de: https://revistas.pucsp.br/index.php/ls/article/view/26678.

Escuelapedia. (n.ed). Toyotismo. recuperado de: http://www.escuelapedia.com/el-toyotismo/.

Foss NJ, K. C. (1995). An exploration of common ground: integrating evolutionary and strategic theories of the firm. Recuperado de: https://link.springer.com/chapter/10.1007/978-14615-2201-0_1.

Gramsci, A. (1973). Americanismo y fordismo. Roma: Riuniti.

Hobsbawm, E. (1988). Industria e imperio. Barcelona: Ariel.

Hoffman, N. (2012). Taylorismo, fordismo y toyotismo. recuperado de: http://nuevasensenanza.blogspot.com/2012/06/taylorismo-fordismo-y-toyotismo.html.

Mayoral, L., \& J., T. (2001). Administración de empresas y metodología de investigación : Aportes para un diálogo y una enseñanza efectivos. recuperado de: http://www.redalyc.org/articulo.oa?id $=401137445003$.

McGregor, D. (1960). El lado humano de las empresas. Mexico. ISBN: 9789701058718: Mc Graw-Hill Interamericana S.A.

México, U. N. (2003). Administración básica I. Facultad de contaduría y administración. recuperado de: http://www.itson.mx/publicaciones/pacioli/Documents/no59/administracion_general/vinc ula_pyme.pdf.

Ponce, A. (1992). Administración moderna. recuperado de: https://books.google.es/books?hl=es\&lr=\&id=TwnmlLyBJIYC\&oi=fnd\&pg=PA2\&dq=A ugustin+Ponce\&ots=yUoiXPuKEf\&sig=6NQbMWSYbzTOwT_4auuLxqGrnvU\#v=onep age $\& \mathrm{q} \& \mathrm{f}=$ false.

Porter, M. (1985). How information gives you competitive advantage. Harvard Business Review.

Porter, M. (2017). El ser competitivo. recuperado de: https://www.casadellibro.com/librosebooks/michael-e-porter/62278.

Sonora, I. T. (2013). La Cultura Organizacional en el siglo XXI: Un estudio bibliométrico en Latinoamérica. recuperado de: www.itson.mx.

Soteldo, H., \& al, e. (2005). Diagnóstico de las condiciones internas para la gestión del talento humano en las Pymes de la región centro-norte del Estado de Sinaloa. recuperado de: http://www.itson.mx/publicaciones/Documents/ciencias-economico/estudiosdeadm. 